for various micro-organisms, the full details of practical importance are given for bacteria only. Different methods of bacterial preservation are presented, freeze-drying being described in every detail from culture inoculation to the final rehydration and recovery testing. There is no doubt that for long storage and convenient transport this is the method of choice for the majority of genera and species. The technical requirements for every bacterial species preserved by freeze-drying in the NCTC are recorded. Anyone seeking a simple and a reliable method of preservation for a particular bacterium should consult this monograph.

The authors have also referred to valuable information from publications throughout the world. Unfortunately, they have not always been so careful when reviewing the work of others as when presenting their own experience. As an example, the authors are wrong to indicate that liquid nitrogen was used for pre-freezing in the freezing-drying machine described by Greiff and Rightsel (pp. 8-9), when actually it was used only for cooling the condenser. Also, when discussing the cryoprotectants there is no reason for referring to the original work on glycerol and not doing the same for dimethylsulphoxide (p. 7).

This book can be recommended to all microbiologists interested in the preservation of micro-organisms.

\title{
V. Damjanovic
}

No. 8. Isolation of salmonellas. By R. W. S. Harvey and T. H. Price. 1975. Pp. iii and 52. $£ 1 \cdot 50$.

In part I of this monograph the "Principles of salmonella isolation " that are discussed include: temperature of incubation; motility enrichment; pre-enrichment, enrichment and plating media; and the effect of sample size.

In part II, entitled " Techniques recommended for different samples", there are nine sections. Only two of these, on human faeces and human blood, are likely to concern the routine diagnostic laboratory in a hospital and they consist of less than two pages of text and three figures. The recommended methods are more thorough than those in common use and would undoubtedly give higher isolation rates. The reliance on direct slide agglutination rather than fermentation tests as a first procedure on non-lactose-fermenting colonies is sound. The other sections concern specimens likely to be dealt with only by public-health or veterinary laboratories: animal faeces, human and animal foodstuffs, sewer swabs, polluted waters, and cultures referred from other laboratories. These sections describe many ingenious dodges for isolating salmonella serotypes from grossly contaminated materials and from specimens that contain many serotypes.

The appendix contains details of media and of glass apparatus that would have delighted Sir Almroth Wright. The section on serological reagents suggests that salmonella agglutinating sera are generally available from the Standards Laboratory in the Central Public Health Laboratory, London. In fact, only the Public Health Laboratory Service is supplied from this source.

The monograph, written in a chatty and anecdotal style, is easy to read.

ROBERT BLOWERS

\section{Thymus factors in immunity}

Edited by H. Friedman. 1975. Annals of the New York Academy of Sciences, vol. 249. New York: New York Academy of Sciences. Pp. 547. US \$39.00 in paperback.

In 1951 Miller showed the importance of the thymus in cell-mediated immunity and antibody production. Despite early experiments in which some of the effects of neonatal thymectomy were reversed by thymus in a millipore chamber, the role of thymus hormones was regarded as unlikely or unimportant. The present work indicates the change in their status that has emerged mainly through the poineer work of Trainin, A. Goldstein and White, and G. Goldstein. 
Although this volume deals with T-cell products and the collaboration of $\mathrm{T}$ and $\mathrm{B}$ cells, its distinctive contribution is on the preparation and properties of thymus hormones. At least some of these hormones are fairly stable as indicated by the use of heat to precipitate irrelevant protein in their preparation. Their properties include the ability to increase the number of $T$ cells, as judged by $E$ rosettes, when added to lymphocytes of patients with immune deficiency affecting $T$ cells, the ability to cause certain bone-marrow cells to acquire thymus surface-antigens, the ability to restore in-vitro graft-versus-host reactions to the spleen of irradiated, neonatally thymectomized and aged mice, the increase of antibody production in irradiated animals and the hastening of antibody production during the neonatal period.

The volume clearly puts thymus hormones on the map and raises the question of their use in man. Many problems, however, remain unsolved. First, is there a whole range of thymus hormones? There is some evidence that thymus hormone limits autoantibody production in NZB mice and may be needed for the maintenance of suppressor $T$ cells. There is other evidence that thymus hormone favours the restoration of antibody production and cell-mediated immunity after irradiation. The relatively slight effect of adult thymectomy in man suggests that there are several thymus hormones with opposing effects. It would be useful to know how many different hormones exist.

Second, most endocrine organs have a complex feedback control of secretion so as to respond to changes in the environment. We now need to know what signals control the release of thumus hormones by the thymus.

Third, it is not clear which thymus hormones act locally, on cells entering the thymus and coming in close contact with thymus epithelial cells, and which normally act systemically. The loss of suppressor cells early after adult thymectomy suggests that this may be one situation in which thymus hormones act systemically.

This book is excellent value for those interested in thymus hormones and should hasten the advance of knowledge of these factors and their application to human disease.

$$
\text { G. L. AsHERSON }
$$

\section{Dermatophytes in human skin, hair and nails}

By J. T. SInski. 1974. Springfield, Illinois, USA: Charles C. Thomas. Pp. xiv and 43; 35 figs. $\$ 7.95$.

This is quite a useful book on the identification of dermatophytes in clinical specimens. However, most of those who do this work will surely already have an " Atlas of medical mycology ", or indeed a general handbook of laboratory techniques, which probably covers the ground adequately.

H. I. WINNER 